\title{
ON THE POSSIBILITY OF SPONTANEOUS CURRENTS IN MESOSCOPIC SYSTEMS
}

\author{
E. Zipper, M. SzopA \\ Instilute of Pliysics, University of Silesia \\ Uniwersytecka 4, 40-007 Katowice, Poland \\ D. Wohlleben, P. Freche and M. Esser \\ II. Physikalische Institut der Universität zu Köln \\ 5000 Köln 41, Germany
}

It is shown that a mesoscopic metallic systcm can exhibit a phase transition to a low temperature state with a spontaneous orbital current if it is sufficiently free of elastic defect scattering. The interaction among the electrons, which is the reason of the phase transition, is of the magnetic origin and it leads to an ordered state of the orbital magnetic moments.

PACS numbers: $71.30 .+h, 72.10 .-\mathrm{d}, 71.10 .+\mathrm{x}$

Quantum persistent currents in mesoscopic non-superconducting rings threaded threaded by a magnetic flux $\phi$ aroused great excitement and controversy in the last years.

They were predicted theoretically [1] some years ago and detected recently in a collection of isolated mesoscopic rings [2].

The flux $\phi$, which drives the persistent current $I(\phi)$, is the sum of the externally applied flux $\phi_{\mathrm{e}}$ and of the flux $\phi_{I}$ from the persistent current itself

$$
\phi=\phi_{\mathrm{e}}+\phi_{r},
$$

where $\phi_{I}=\mathcal{L} I$ and $\mathcal{L}$ is the self-inductance of the system.

Most theoretical discussions neglect the second term, which is justified by the experimental structures realized so far.

IIowever, we want to investigate the case when the current $I$ in a sample is so large that $\phi_{I}$ cannot be neglected. In a very clean system (low level of elastic defect scattering), at small $\phi_{\mathrm{e}}$ and at sufficiently low temperature, $\phi_{I}$ can become of the order of $\phi_{\mathrm{e}}$ or even larger. The formula for the current $I(\phi)$ is a nonlincar function of $\phi$ (see later) and because $\phi=\phi_{\mathrm{e}}+\mathcal{L} I$, there is a possibility of the existence of solutions with finite $I$ at $\phi_{e}=0$, i.e. the solutions with spontaneous orbital currents.

In this paper we show that in the ballistic regime, i.e. when the elastic mean free path is larger than the circumference of the ring, a mesoscopic system can make 
a transition from a high temperature state wilh zero current to a low temperature state with a persistent current in the absence of an external field. In other words, wc show that the ground state of such a system is in general a flux phase state [3], i.e. a state with a spontaneous magnetic flux generated by a spontancous orbital current.

The transition temperature and the spontaneous persistent current depend strongly on the geometry of the ring and of the Fermi surface.

To present the idea we perform the model calculations for a system wilh a nearly flat Fermi surface (FS) perpendicular to the momentum direction $p_{\varphi}$, i.e. a nearly one-dimensional electron gas. This is the most favorable case because the current $I$ is then the strongest.

Such a material can be obtained for instance if one winds $M$ metallic chains (certain organic molecules) in a very weak transverse electrical contact around a mesoscopic cylinder of a radius $R$ (we use cylindrical coordinates $(R, z, \varphi)$. Thus we can visualize our system of $N$ electrons as a set of $M$ current carrying channels.

We work in a gauge for the vector potential in which the field does not appear explicitly in the Iramiltonian but enters the calculation via the flux-modified boundary conditions [4]

$$
\psi(2 \pi)=\mathrm{e}^{\mathrm{i} 2 \pi \phi / \phi_{0}} \psi(0), \quad \frac{\mathrm{d} \psi}{\mathrm{d} \varphi}(2 \pi)=\mathrm{e}^{\mathrm{i} 2 \pi \phi / \phi_{0}} \frac{\mathrm{d} \psi}{\mathrm{d} \varphi}(0) .
$$

In a magnetic field $B \| z$ the electrons fill the quantized energy levels [4]

$$
\varepsilon_{n}=\frac{\hbar^{2}}{2 m R^{2}}\left(n-\frac{\phi}{\phi_{0}}\right)^{2}, \quad n=0, \pm 1, \pm 2, \ldots,
$$

where $n$ parametrizes the angular momentum in $\varphi$ direction, $\phi_{0}=h / e$. To each $\varepsilon_{n}$ there corresponds a current

$$
i_{n} \equiv-\frac{\partial \varepsilon_{n}}{\partial \phi}=\frac{e \hbar}{2 \pi m R^{2}}\left(n-\frac{\phi}{\phi_{0}}\right)
$$

and the total current $I$ is

$$
I=\sum_{n} i_{n} f_{\mathrm{FD}}\left(\varepsilon_{n}\right)
$$

where $f_{\mathrm{FD}}\left(\varepsilon_{n}\right)$ is the Fermi-Dirac distribution function.

Equation (1) means that each electron moves not only in the external magnetic field but also in the magnetic field produced by all other electrons. Each current loop is equivalent to the magnetic dipole. The magnetic dipole-dipole interaction is taken here in the mean field approximation. In other words, because of electromagnetic coupling each electron adds its own contribution to the total flux and sees the flux of all others, except its own. This works only for many electron systems and only for $M \gg 1$.

The system has a set of quantum size energy gaps - the gap at the FS is $\Delta=\left(\hbar^{2} / m R^{2}\right) n_{\mathrm{F}}$, where $n_{\mathrm{F}}$ numbers the last occupied state. For $N \approx 10^{7}$ electrons moving in $M \approx 10^{4}$ rings with a radius $R \approx 400$ we get $\Delta \approx 300 \mathrm{~K}$.

The total energy is periodic in $\phi_{0}$, the current at $k T \ll \Delta$ is persistent because of the gap [4]. It turns out that the properties of the system depend strongly on the number of elcctrons. 
In order to understand how the flux phase can be created in the mesoscopic system let us calculate minimum of the free energy $F=E-T S$ of the system at $\phi_{\mathrm{e}}=0$ and $T=0$ as a function of $\phi[5]$. Assuming that the number $N$ of electrons is constant we consider two cases: $N / M$ odd and $N / M$ even. In the first case we can assume that $N=M\left(2 n_{F}+1\right)$, i.e. to each channel there correspond $2 n_{F}+1$ electrons occupying symmetrically $n=0, \pm 1, \ldots, \pm n_{F}$ states. In this case the free energy is

$$
F(\phi)=E(\phi)=M \sum_{n=-\infty}^{\infty} \varepsilon_{n} f_{\mathrm{FD}}\left(\varepsilon_{n}\right)+\frac{1}{2} \mathcal{L} I^{2},
$$

where the Fermi factor $f_{\mathrm{FD}}\left(\varepsilon_{n}\right)$ is a step like a function with the chemical potential $\mu=\varepsilon_{n_{\mathrm{F}}+1 / 2}$. After a little algebra we find that

$$
F(\phi)=F(0)+\left(\frac{\hbar^{2} N}{2 m R^{2} \phi_{0}^{2}}+\frac{1}{2 \mathcal{L}}\right) \phi^{2}>F(0), \quad|\phi|<\phi_{0} / 2 .
$$

The free energy of the system with $N / M$ odd increases quadratically with $\phi$, the terms linear in $\phi$ cancel because of equal occupation of $\pm n$ states and therefore the ground state is a state with zero flux and zero current.

In the second case when $N=M 2 n_{F}$ in each channel we have one odd electron, which must be placed at $\pm n_{F}$. Depending on $\phi$ it will occupy $+n_{F}$ (for $\phi>0),-n_{\mathrm{F}}($ for $\phi<0)$ or both states (for $\phi=0$ ). The free energy is in this case

$$
F(\phi)=E(0)+\Delta M\left[-\frac{|\phi|}{\phi_{0}}+\left(\frac{\phi}{\phi_{0}}\right)^{2}\right]+\frac{\phi^{2}}{2 \mathcal{L}}, \quad|\phi|<\phi_{0} / 2,
$$

i.e. apart from the quadratic term it contains now the term which decreases with increasing $|\phi| . F$ has then two minima corresponding to the spontaneous flux at $\phi_{\mathrm{e}}=0$

$$
\frac{\phi_{\mathrm{sp}}}{\phi_{0}}= \pm \frac{1}{2+\phi_{0}^{2} / M \Delta \mathcal{C}} .
$$

This flux is approaching $\phi_{0} / 2$ in the limit of large $M$ and zero in the opposite limit. Inserting (9) to (8) we get the free energy of the ground state

$$
F\left(\phi_{\mathrm{sp}}\right)=F(0)-\frac{(\Delta M)^{2}}{\Delta M+\phi_{0}^{2} / 2 \mathcal{L}}<F(0),
$$

i.e. the energy of the state with a spontaneous flux is lower than the energy of the electrons at zero current (equal partial occupation of $\pm n_{F}$ states). A ground state with a spontaneous flux may be called a flux phase state [3]. In this state we have a spontaneous orbital current

$$
I= \pm \frac{\phi_{0} \Delta M}{\phi_{0}^{2}+2 \mathcal{L} \Delta M}
$$

which remains persistent also for $0<k T \ll \Delta$. The current (11) approaches $I_{0} / 2 \equiv \phi_{0} / 2 \mathcal{L}$ in the limit of large $M$ and zero in the opposite limit. We neglect the contribution due to spin because, if the spin and orbital moments of not completely filled shells in atoms are comparable, in mesoscopic systems the latter dominate. In short, at $T=0$ and $\phi_{\mathrm{e}}=0$ the mesoscopic system considered here carrics in 
general a spontaneous flux coming from the uncompensated orbital currents. This spontaneous current is a ground state property it will vanish at temperatures in the neighborhood of

$$
k T_{\mathrm{c}} \approx \frac{(M \Delta)^{2}}{\left(M \Delta+\phi_{0}^{2} / 2 \mathcal{L}\right)}
$$

see Eq. (10).

Let us investigate now the temperature dependence of the free energy of the system [6]

$$
\begin{gathered}
F(T, \phi, N)=-k T M \sum_{n} \ln \left[1+\exp \left\{-\left[\varepsilon_{n}(\phi)-\mu(\phi, N, T)\right] / k T\right\}\right] \\
+N \mu(T, \phi, N)+\left(\phi-\phi_{\mathrm{e}}\right)^{2} / 2 \mathcal{L},
\end{gathered}
$$

where the chemical potential $\mu$ is calculated from the condition

$$
N=M \sum_{n} \frac{1}{1+\exp \left\{\left[\varepsilon_{n}(\phi)-\mu(N, T, \phi)\right] / k T\right\}} .
$$

The numerical calculations of $F(T, \phi, N)$ at $\phi_{\mathrm{e}}=0$ are presented in Fig. 1 for $N / M$ odd and in Fig. 2 for $N / M$ even.

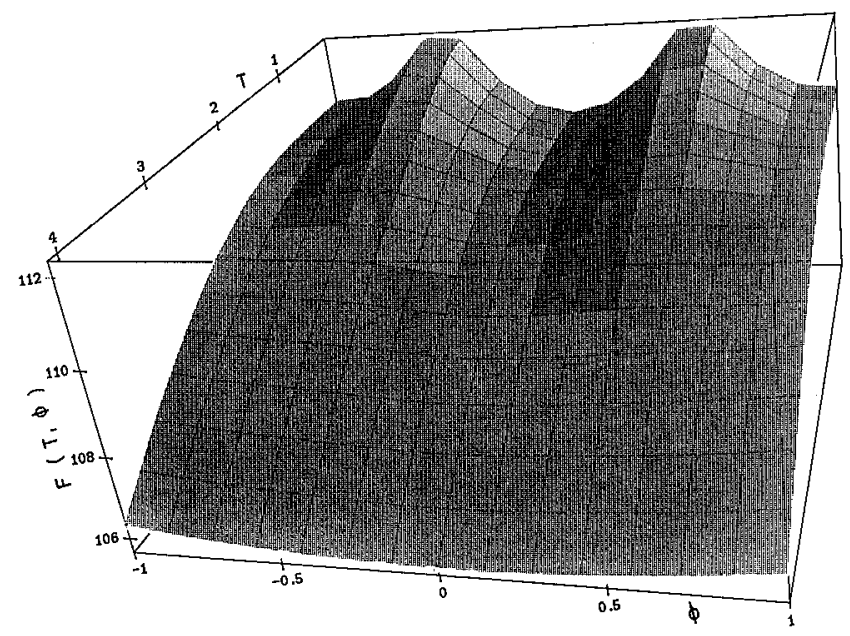

Fig. 1. The free energy of a system, where every ring carrics an odd number of spin-less electrons $\left(M_{\mathrm{e}} / M=0, M_{\mathrm{e}}\right.$ is the number of rings, which carry an even number of electrons), as a function of the flux $\phi$ and temperature $T$. The minima of $F$ lie always at $\phi=0$ (and some multiples of $\phi_{0}$ ).

In the case of $N / M$ odd the free energy always has an absolute minimum at $\phi=0$. It has also some local minima at $\phi=n \phi_{0}$.

The situation looks different at $N / M$ even. At low $T$ we have two symmetric minima of $F$, corresponding at $T=0$, to spontaneous fluxes given by Eq. (9), separated by a maximum at $\phi=0$. 


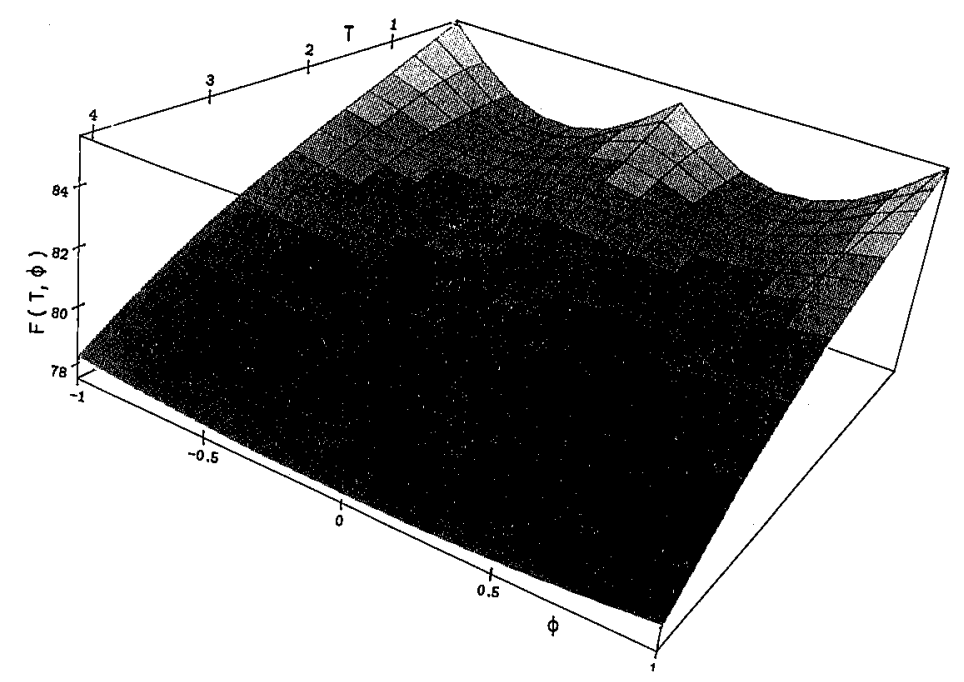

Fig. 2. The free energy of a system, where every ring carrics an even number of spin-less electrons $\left(M_{c} / M=1\right)$, as a function of the flux $\phi$ and temperature $T$. At $T>T_{\mathrm{c}}$ the minima of $F$ lie at $\phi=0$ (and some integer multiples of $\phi_{0}$ ). At $T<T_{c}$ two symmetrical minima of $F$ at $\phi= \pm \phi_{\mathrm{sp}}(=0)$ develop.

With increasing $T$ the minima become shallower with a simultaneous decrease in $\phi$. At a certain temperature $T_{c}, F(\phi)$ has the inflection point and at $T \geq T_{\mathrm{c}}$ we have only one minimum at $\phi=0$.

We see that there exists a certain temperature $T_{c}$, below which the system spontaneously breaks the symmetry, i.e. it comes to the state with the spontaneous magnetic flux $\phi$. In other words, for $T$ above $T_{\mathrm{c}}, F$ is simply a parabolic shaped curve, with a well-defined minimum. $A s T$ approaches $T_{\mathfrak{c}}$, the minimum becomes shallower. When $T$ passes through $T_{\mathrm{c}}$, the minimum at $\phi=0$ becomes a local maximum and the minima develop at $\phi= \pm \phi_{\mathrm{sp}}$. erties:

$\phi_{\mathrm{sp}}$ can be treated as an order parameter because it lias the following prop-

1. In the disordered phase at $T>T_{\mathrm{c}} \phi_{\mathrm{sp}}=0$, whereas in the ordered phase at $T<T_{\mathrm{c}} \phi_{\mathrm{sp}} \neq 0$.

2. $\phi_{\mathrm{sp}}$ tends continuously to zero in the ordered phase if $T \rightarrow T_{\mathrm{c}}$.

3. Below $T_{\mathrm{c}}$ the order parameter is not fully determined by the external conditions, but on an equal footing it can have two values minimizing the free energy.

Let us expand the free energy $F$ given by Eq. (13) in a series of $\phi$ for $\phi_{\mathrm{e}}=0$, at temperatures close to $T_{c}$, and look for the expansion coefficients

$$
F=F_{0}+\kappa \phi+a \phi^{2}+\gamma \phi^{3}+b \phi^{4}+\ldots,
$$

where $F_{0}$ is the free energy in the disordered phase. It follows from the symmetry properties of $\mu(T, \phi, N)$ that $\left.\frac{\partial \mu}{\partial \phi}\right|_{\phi=0}=0$. Using this we can calculate the $\kappa$ and $\gamma$ 


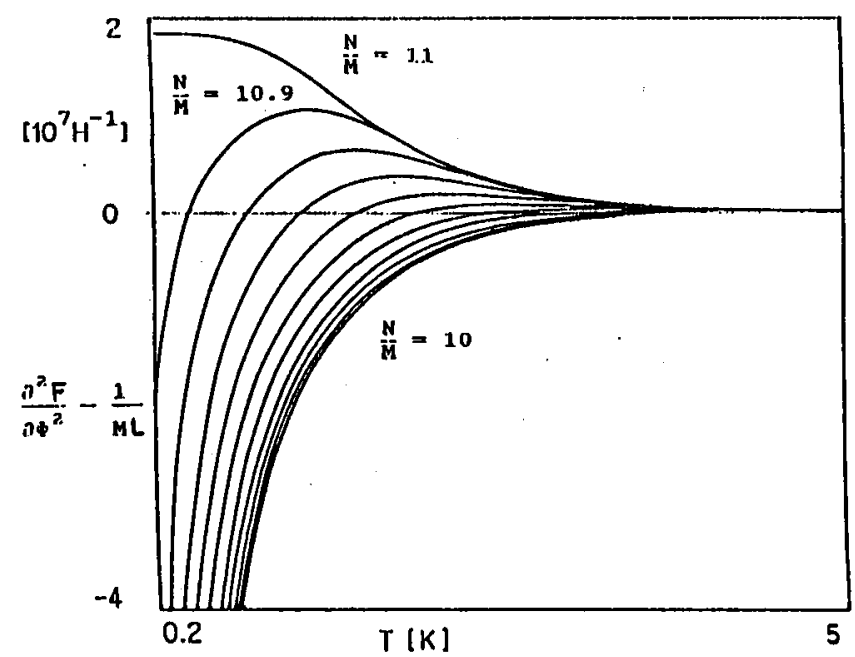

Fig. 3. The value of $\partial^{2} F / \partial \phi^{2}-1 / M L$ at $\phi=0$ as a function of temperature in zero external magnetic ficld for the ratio $N / M_{2}$ changing from 10 to 11 and $R=400$ $\AA$. The temperature, at which $\partial^{2} F / \partial \phi^{2}$ is equal to zero, defines the transition from the high temperature phase, with zero current, to the low temperature phase with the spontaneous flux. For large $M L(1 / M L=0)$ the transition temperature is determined by the zero of $y$-axis whereas for finite $M L$ it corresponds to $-1 / M L$ at this axis.

coefficients. We get

$$
\kappa=\left.\frac{\partial F}{\partial \phi}\right|_{\phi=0}=0, \quad \gamma=\left.\frac{\partial^{3} F}{\partial \phi^{3}}\right|_{\phi=0}=0 .
$$

Figure 3 shows the temperature dependence of $a=\left.\frac{\partial^{2} F}{\partial \phi^{2}}\right|_{\phi=0}$,

$$
a(T)=\left.\frac{1}{2} \frac{\partial^{2} F}{\partial \phi^{2}}\right|_{\phi=0}=\frac{1}{M \mathcal{L}} \frac{2 \alpha}{\phi_{0}^{2}}\left\{\frac{N}{M}-\alpha \beta \sum_{n \in N} \frac{n^{2}}{1+\cosh \left[\left.\beta\left(\varepsilon_{n}-\mu\right)\right|_{\phi=0}\right]}\right\} .
$$

We see that $a\left(T_{\mathrm{c}}\right)=0, a\left(T>T_{\mathrm{c}}\right)>0, a\left(T<T_{\mathrm{c}}\right)<0$. In the neighborhood of $T_{\mathrm{c}}$ $a$ can be approximated by a linear relation

$$
a(T)=A\left(T-T_{\mathrm{c}}\right), \quad A>0 .
$$

The numerical calculations of $b=\left.\frac{\partial^{4} F}{\partial \phi^{4}}\right|_{\phi=0}$ show that it is roughly constant at $T$ close to $T_{\mathrm{c}}$. Thus we see that expansion (15) is a Ginzburg-Landau free energy

$$
F=F_{0}+A\left(T-T_{\mathrm{c}}\right) \phi^{2}+b \phi^{4}
$$

which corresponds to a second order phase transition. The transition temperature can be calculated from the equation $a(T)=0$. Thus the energy $(13)$ at $T \approx T_{\mathrm{c}}$ describes the phase transition from the high temperature phase with $\phi=0$ to the low temperature phase with $\phi \neq 0$. 
The value of the spontaneous current and hence the spontaneous flux can be calculated in a direct way using Eq. (5) for the current. Let us consider the case with a spontaneous flux, i.e. when $N / M$ is even. For the convenience of numerical calculations we bring this equation to the form, in which the summation over the whole spectrum of states $\varepsilon_{n}(-\infty<n<+\infty)$, is well approximated by the summation over $s$ states in the neighborhood of the Fermi lovel $n_{F}-s<|n|<$ $n_{\mathrm{F}}+s$

$$
I(\phi)=c N \sum_{k=1}^{s}\left[\frac{\operatorname{sh}\left[\frac{c N}{2 T}\left(\phi-\frac{\phi_{0}}{2}\right)\right]}{2 \operatorname{ch}\left[\frac{c N}{4 T}\left(\phi+\frac{2 k+2}{2}\right)\right] \operatorname{ch}\left[\frac{c N}{4 T}\left(\phi-\phi_{0}-\frac{2 k+1}{2}\right)\right]}-\frac{\phi}{\phi_{0}}-\frac{1}{2}\right],
$$

where $c=e h / m \mathcal{L}^{2}$. On the other hand we want to have a current that corresponds to the minimum of the free encrgy (13). It occurs that the condition for that extremum $\left(\frac{\partial F}{\partial \phi}=0\right)$ yields, for fixed $T, N$ and $\phi_{\mathrm{e}}$, Eq. (1) which we now rewrite in a form

$$
I(\phi)=\frac{1}{\mathcal{L}}\left(\phi-\phi_{\mathrm{e}}\right)
$$

The graphical solutions of those two self-consistent equations for zero external field $\left(\phi_{e}=0\right)$ are presented in Fig. 4. The intersection of the two curves give the values of the spontaneous current.

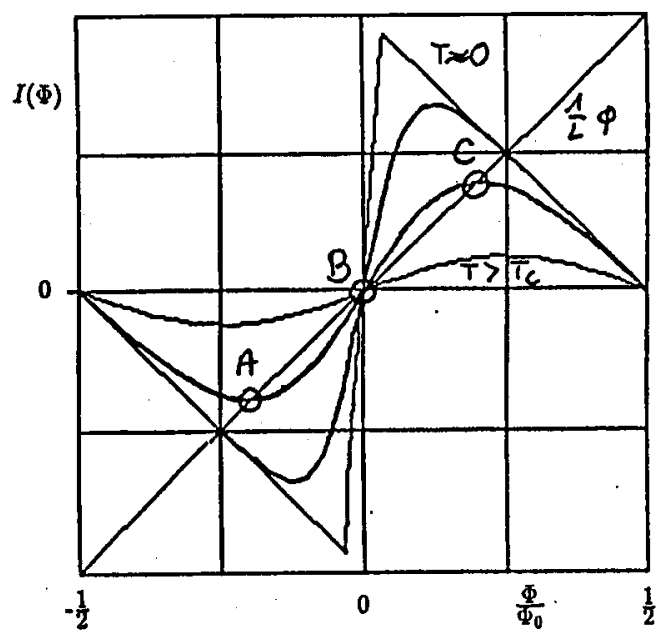

Fig. 4. Graphical representation of the self-consistency equation $I(\phi)=\left(\phi-\phi_{\text {ext }}\right) / L$ for $\phi_{\text {ext }}=0$. Trivial solution at $T>T_{\mathrm{c}}(B)$; nontrivial solutions at $T>T_{\mathrm{c}}(A$ and $C)$.

At high $T$ the only solution is $I=0$. Al $T<T_{\mathrm{c}},\left(T_{\mathrm{c}}\right.$ is a temperature at which the curve of Eq. (21) is tangent to the curve of Eq. (20)) the two new solutions appear. They correspond to the spontaneous orbital currents (spontaneous orbital magnetic moments) and they are at the minimum of the free energy.

The interaction among the electrons which is the reason of the phase transition to the low temperature ordered phase is here of the magnetic origin, namely of the dipole-dipole type. 
It resembles the ferromagnetic ordered state due to the exchange interaction among spins in ferromagnets. Ilowever, there are some important differences. One of them is the following. If we apply the external magnetic field to the ferromagnet in the ordered state we get a paramagnetic reaction. In our case the application of $\phi_{\mathrm{e}}$ always gives a diamagnetic reaction at low $T$. One can see it from the formula $(21)$ and Fig. 4 if we take $\phi_{\mathrm{e}} \neq 0$. The straight line $I(\phi)=(1 / \mathcal{L})\left(\phi-\phi_{\mathrm{e}}\right)$ moves then down and the cross-section will be at lower $I$ (lower spontaneous moment) and at low $T$.

The phenomenon presented by us resembles strongly the early efforts to explain ferromagnetism by the magnetic interaction (see e.g. [7]).

The spontaneous flux as a function of temperature for ten equally spaced values of $N: 2 n_{F} M \leq N \leq\left(2 n_{F}+1\right) M$ is presented in Fig. 5 . We observe the

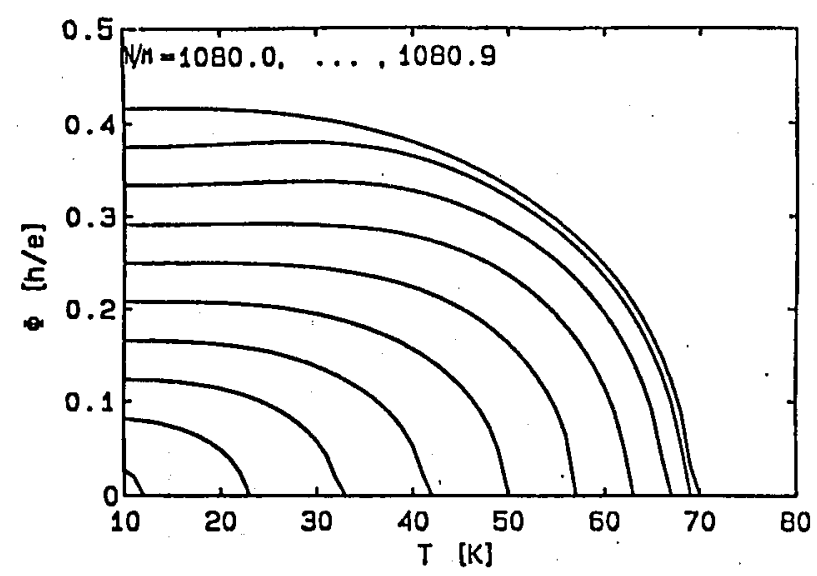

Fig. 5. The spontaneous flux as a function of temperature for ten equally spaced values of $M_{\mathrm{e}} / M$. At high temperatures $\phi_{\mathrm{sp}}$ is zero for all $M_{\mathrm{e}}$ and there is no flux down to the lowest $T$ for $M_{\mathrm{e}}=0$. For $M_{\mathrm{e}}>0$ with decreasing $T$ a spontaneous flux appears at a certain temperature $T_{\mathrm{c}}$.

following features: At high $T \phi_{\mathrm{sp}}=0$ at all $N / M$ and there is no flux down to the lowest $T$ for odd $N / M$. For $N / M$ even with decreasing $T$ a spontaneous flux appears at a certain temperature $T_{\mathrm{c}}$ and increases up to the value which is roughly proportional to the deviation $\delta(0<\delta<1)$ of $N / M$ from exactly odd

$$
\phi_{\mathrm{sp}}\left[N=M\left(2 n_{\mathrm{F}}+1-\delta\right)\right] \simeq \phi_{\mathrm{sp}}\left[N=M 2 n_{\mathrm{F}}\right] \delta .
$$

The above considerations show that while depending on the $N / M$ ratio one can get almost any value for $\phi_{\mathrm{sp}}$ but in discrete steps. The flux, which minimizes the total energy, now enters the boundary conditions via Eq. (2). A flux $\phi \neq n \phi_{0}$ is mathematically equivalent to a change in the boundary conditions. In other words, the spontaneous flux can shift the phase of the single electron states by any value between $0<|\delta \chi| \equiv 2 \pi\left|\phi_{\mathrm{sp}}\right| / \phi_{0}<\pi$, depending on the parameters of the system.

This distinguishes flux phase states from the current carrying states of certain 
atoms with partially filled shells. In such atoms the self-inductance and the number of channels are so small that $\phi_{\text {sp }} \ll \phi_{0}$, i.e. always $|\delta \chi| \ll \pi$.

The above mean field results will be reduced by fluctuations. Below $T_{\mathrm{c}}$ the system is in a minimum of a mean field $F(\phi)$, at the finite spontaneous flux $\phi_{\text {sp }}$. The difference $\Delta F \equiv F(0)-F\left(\phi_{\mathrm{sp}}\right)$ is $\Delta F \cong\left|\left(\phi_{\mathrm{sp}}^{2} / 2\right) \mathrm{d}^{2} F / \mathrm{d} \phi^{2}\right|_{\phi=0}=\phi_{\mathrm{sp}}^{2} / 4 \mathcal{L}$. If the energy $\varepsilon_{\mathrm{f}}$ driving the fluctuations is small $\left(\varepsilon_{\mathfrak{f}} \ll \Delta F\right)$, the resulting Gaussian fluctuations $\Delta \phi$ of the flux will be given by

$$
\{\Delta \phi\}^{2}=\frac{2 \varepsilon_{\mathrm{f}}}{\mathrm{d}^{2} F / \mathrm{d} \phi^{2}}, \quad\left(\{\Delta \phi\}^{2} \approx 2 \varepsilon_{\mathrm{f}} \mathcal{L} \text { near } T=0\right) .
$$

In the case of the thermal current fluctuations, driven e.g. by inelastic electronphonon scattering, we lave $\varepsilon_{\mathrm{f}} \leq k_{\mathrm{B}} T / 2$, i.e. $\Delta \phi(T) \leq\left(\mathcal{L} k_{\mathrm{B}} T\right)^{1 / 2}$. For our ring

$$
\Delta \phi(T) \leq \phi_{0}\left\{10^{-4}[T(K)]^{1 / 2}\right\},
$$

i.e. $\Delta \phi(T) \ll \phi(T)$ except close to $T_{\mathrm{c}}$ (see Fig. 5). Near $T=0$ quantum fluctuations are driven by the zero point motion of the electromagnetic field. A zero-point photon with the energy $h \nu=h c / \lambda=2 \Delta$ can cause virtual electron hole excitations across the gap $2 \Delta=\varepsilon\left(n_{F}+1\right)-\varepsilon\left(n_{F}\right)$. Quantum fluctuations of the ring current will then be driven by $\varepsilon_{\mathrm{f}}=2 \Delta(R / \lambda)^{3} \cong 2 \Delta\left(2 v_{\mathrm{F}} / c\right)^{3},(\lambda \gg R)$. In our case $\varepsilon_{\mathrm{f}} \cong 2 \Delta(R / \lambda)^{3} \cong 30 \mu \mathrm{K}$. The reductions of the mean field $T_{\mathrm{c}}$ 's by thermal and quantum fluctuations will therefore remain small.

Summarising we have shown that a sufficiently pure mesoscopic metallic system can exhibit a phase transition to a low temperature state with a persistent current in zero external flux due to the magnetic coupling of electrons. We have estimated the dependence of the spontaneous orbital current (flux) on the number of particles and on temperature.

This work was supported in part by the Polish Ministry of Science, the grant of the the Committee for Scientific Research (KBN) 1770/2/91 and by SFB 341 of the Deutsche Forschungsgemeinschaft.

\section{References}

[1] M. Buttiker, Y. Innry, R. Landauer, Phys. Lett. A 96, 365 (1983).

[2] L.P. Levy, G. Dolan, J. Dunsmuir, II. Bouchiat, Phys. Rev. Lett. 64, 2074 (1990).

[3] V. Kalmaeyer, R.B. Laughlin, Phys. Rev. Lett. 59, 2095 (1987).

[4] H.F. Cheung, Y. Gefen, E.K. Riedel, W.II. Shih, Phys. Rev. B 37, 6050 (1988); D. Wohlleben, J. Less-Common Met. 138, 11 (1988); M. Szopa, E. Zipper, in: Physics and Materials Science of High Temperature Superconductors, Eds. R. Kossowski, S. Methfessel, D. Wollleben, NATO ASI Series, Vol. 181, Kluwer, Amsterdam 1990, p. 139.

[5] M. Szopa, D. Wollleben, E. Zipper, Phys. Lett A 160, 271 (1991).

[6] D. Wohlleben, M. Esser, P. Freche, E. Zipper, M. Szopa, Phys. Rev. Lett. 66, 3191 (1991).

[7] R.P. Feynman, R.B. Leighton, M. Sands, The Feynman Lectures on Physics, Vol 2, Addison-Wesley Publ. Co. Inc., London 1964. 